\title{
A note on sequences determined by a recurrence relation
}

\author{
By C. E. Walsh, Dublin.
}

(Received 12th May, 1932. Read 4th June, 1932.)

$\S 1$. Copson and Ferrar have proved the following theorem ${ }^{1}$ :

If $q_{n}=\rho_{n}+i \sigma_{n}, \lim \rho_{n}=h>0, \Sigma\left(1 / p_{n}\right)$ is a divergent series of positive terms, and $\left(p_{n}+q_{n}\right) u_{n}-p_{n} u_{n-1}=y_{n}$ always, where $y_{n} \rightarrow 0$, then $u_{n} \rightarrow 0$.

Let $p_{n} /\left(p_{n}+q_{n}\right)$ and $1 /\left(p_{n}+q_{n}\right)$ be denoted respectively by $a_{n}$ and $c_{n} ;$ then

Here we have

$$
u_{n}=a_{n} u_{n-1}+c_{n} y_{n}
$$

$$
\left|a_{n}\right| \leqq \frac{p_{n}}{p_{n}+h}<1 ; \quad\left|c_{n}\right| \leqq-\frac{1}{p_{n}+h} \leqq \frac{1}{h}\left(1-\left|a_{n}\right|\right) .
$$

Further, since $\Sigma\left(1 / p_{n}\right)$ is a divergent series of positive terms,

$$
\prod_{r=1}^{n} a_{r} \rightarrow 0, \quad \text { as } n \rightarrow \infty \text {. }
$$

Thus the theorem of Copson and Ferrar is included in the following:

If $u_{n}=a_{n} u_{n-1}+c_{n} y_{n}$, where $\mid a_{n}<1$ always, $\prod_{i=1}^{n} a_{r}$ tends to zero, $\left|c_{n}\right| \leqq K\left(\mathbf{l}-\left|a_{n}\right|\right), K$ being fixed, and $y_{n} \rightarrow 0$, then $u_{n} \rightarrow 0$.

A proof of this can be obtained by putting $b_{n}=0$ in Theorem I which occurs later in this paper. The latter concerns sequences $\left(u_{n}\right)$ obtained from a recurrence relation of the form

$$
u_{n}=a_{n} u_{n-1}+b_{n} u_{n-2}+c_{n} \theta_{n},
$$

where $\theta_{n} \rightarrow 0$.

$\S 2$. Supposing equations (1) to have been solved for $u_{n}$, we derive an equation of the form

$$
u_{n}=A_{n}^{n} \theta_{n}+A_{n}^{n-1} \theta_{n-1}+\ldots+A_{n}^{r} \theta_{r}+\ldots+A_{n}^{1} \theta_{1}+B_{n} .
$$

Clearly $A_{n}^{n}=c_{n}$. The following lemma enables us to set bounds to the other coefficients, $A_{n}^{n-1}$, etc., and to $B_{n}$.

1 Copson and Ferrar, Journal London Math. Soc., 4 (1929), 258-264. See also Izumi, Tohoti Math. J., 33 (1931), 181-186. Further references will be found in these papers. 
Lemma. If $\beta_{n}$ be determined by the equations $\beta_{1}=l_{1} \beta_{0}, \beta_{r}=l_{r} \beta_{r-1}+m_{r} \beta_{r-2}$, then

$$
\left|\beta_{r}\right| \leqq\left|\beta_{0} l_{r}\right| \prod_{n=1}^{r-1}\left\{\left|l_{n}\right|+\left|\frac{m_{n+1}}{l_{n+1}}\right|\right\} .
$$

The lemma is obvious when $r=2$. Suppose that it is true for $2,3, \ldots, r-1$. Then

$$
\begin{aligned}
\left|\beta_{r-2}\right| & \leqq\left|\beta_{0} l_{r-2}\right| \prod_{n=1}^{r-3}\left\{\left|l_{n}\right|+\left|\frac{m_{n+1}}{l_{n+1}}\right|\right\} \\
& \leqq\left|\beta_{0}\right| \prod_{n=1}^{r-2}\left\{\left|l_{n}\right|+\left|\frac{m_{n+1}}{l_{n+1}}\right|\right\},
\end{aligned}
$$

so that

$$
\begin{aligned}
\beta_{r} \mid & \leqq\left|l_{r} \beta_{r-1}\right|+\left|m_{r} \cdot \beta_{r-2}\right| \\
& \leqq\left|\beta_{0} l_{r}\right| \prod_{n=1}^{r-1}\left\{\left|l_{n}\right|+\left|\frac{m_{n+1}}{l_{n+1}}\right|\right\} .
\end{aligned}
$$

Thus the result holds good for all values of $r$.

The coefficients $A_{n}^{r}$ in (2) are determined, in virtue of (1), by the equations $A_{r}^{r}=c_{r}, \quad A_{r+1}^{r}=a_{r+1} c_{r}, \quad A_{n}^{r}=a_{n} A_{i-1}^{r}+b_{n} A_{n-2}^{r}$.

Writing $\beta_{0}=A_{r}^{r}=c_{r}, \quad \beta_{3}=A_{r+s}^{r}, \quad l_{s}=a_{r+s}, \quad m_{s}=b_{r+s}$ in the above lemma, it follows that

that is

$$
\left|A_{n}^{r}\right| \leqq\left|a_{n} c_{r}\right| \prod_{s=r+1}^{n-1}\left\{\left|a_{s}\right|+\left|\begin{array}{c}
b_{s+1} \\
a_{s+1}
\end{array}\right|\right\},
$$

and similarly

$$
\left|A_{n}^{r}\right| \leqq\left|c_{r} \prod_{s=r+1}^{n}\right| a_{s} \mid \prod_{s=r+1}^{n-1}\left\{1+\frac{\left|b_{s+1}\right|}{\left|a_{s} a_{s+1}\right|}\right\}
$$

$$
\left|B_{n}\right| \leqq B \prod_{s=1}^{n}\left|a_{s}\right| \prod_{s=1}^{n-1}\left\{1+\frac{\left|b_{s+1}\right|}{\left|a_{s} a_{s+1}\right|}\right\},
$$

$B$ being a constant.

$\S 3$. Now let $u_{n}$, given by equations (1), be expressed as in (2); then certain results connected with its convergence can be obtained. Our mode of proof uses a well known theorem, due to Toeplitz, ${ }^{1}$ namely, that if

$$
u_{n}=A_{n}^{n} \theta_{n}+A_{n}^{n-1} \theta_{n-1}+\ldots+A_{n}^{1} \theta_{1},
$$

where $\theta_{n} \rightarrow 0$, and $A_{n}^{r} \rightarrow 0$ as $n \rightarrow \infty$, for a fixed $r$, while $\sum_{r=1}^{n}\left|A_{n}^{r}\right|<K(a$ constant) then $u_{n} \rightarrow 0$.

${ }^{1}$ K. Knopp, "Theory and Application of Infinite Series" (1928), 72. 
$\begin{aligned} \text { Theorem I. Let } & \text { (i) } \sum_{n=2}^{\infty} \frac{\left|b_{n}\right|}{\left|a_{n} a_{n-1}\right|} \text { converge; } \\ & \text { (ii) }\left|a_{n}\right|<1 \quad \text { for all } n ; \\ & \text { (iii) } \prod_{r=1}^{n} a_{r} \rightarrow 0 \quad \text { as } n \rightarrow \infty ; \\ & \text { (iv) }\left|c_{n}\right| \leqq K\left(1-\left|a_{n}\right|\right), \text { where } K \text { is fixed. }\end{aligned}$

Then if

where

$$
u_{n}=a_{n} u_{n-1}+b_{n} u_{n-2}+c_{n} \theta_{n}
$$

$$
\lim _{n \rightarrow \infty} \theta_{n}=0
$$

then

$$
\lim _{n \rightarrow \infty} u_{n}=0 \text {. }
$$

For, by (3), (i) and (iv), we have

$$
\left|A_{n}^{r}<L\left(1-\mid a_{r}\right)\right| \prod_{s=r+1}^{n}\left|a_{s}\right|,
$$

where $L$ is a constant; for fixed $r, A_{n}^{r} \rightarrow 0$ as $n \rightarrow \infty$, in virtue of (iii). Also

$$
\sum_{r=1}^{n}\left|A_{n}^{r}\right|<L
$$

Again, in virtue of (i) and (iii), $B_{n}$ tends to zero.

Thus we may apply Toeplitz's Theorem to (2), and conclude that $u_{n}$ tends to zero.

$\S 4$. Further similar results may be obtained immediately by the use of the lemma. In particular, the following are of some interest.

Theorem II. Let conditions (ii) and (iii) of the Theorem still hold. Replace (i) and (iv) by

$$
\begin{aligned}
& \left|b_{n}\right|<k\left|a_{n} a_{n-1}\right|, \\
& c_{n} \mid<K\left(\mathbf{1}-\mid a_{n}\right)(\mathbf{1}+k)^{n},
\end{aligned}
$$

where $k, K$ are constants. Then it follows that

$$
(1+k)^{-n} u_{n} \rightarrow 0 \text {. }
$$

For the conditions of Toeplitz's theorem are satisfied, since

and

$$
\begin{aligned}
& (1+k)^{-n}\left|A_{n}^{r}\right|<K\left(1-a_{r} \mid\right) \prod_{s=r+1}^{n}\left|a_{s}\right| \\
& (1+k)^{-n} B_{n} \rightarrow 0 .
\end{aligned}
$$


Theorem III. If the conditions (i)-(iv) of the Theorem be replaced by

$k, l, K$ being constants, then

$$
\begin{aligned}
& 0<k \leqq \prod_{r=1}^{n}\left|a_{r}\right| \leqq l, \\
&\left|c_{n}\right|<K, \\
& \sum_{n=1}^{\infty} \mid \frac{b_{n} \mid}{\left|a_{n}\right|} \text { convergent },
\end{aligned}
$$

$$
u_{n} / n \rightarrow 0 .
$$

In this case $\left|a_{n}\right| \geqq k / l$, so that the series

$$
\Sigma \frac{b_{n} \mid}{\left|a_{n} a_{n-1}\right|}
$$

is convergent. It can easily be shewn that $\left\{A_{n}^{\tau}|,| B_{n} \mid\right.$ are bounded, so that the result follows at once from Toeplitz's Theorem. It is evident that this is still true if we only assume

and

$$
\left|a_{n}\right| \leqq 1, \quad\left|c_{n}\right|<K
$$

$$
\sum_{n=1}^{\infty} \frac{\left|b_{n}\right|}{\left|a_{n}\right|} \text { convergent. }
$$

Theorem IV. If the conditions (i)-(iv) of the Theorem be replaced by

where $k, l, K$ are constants, then

$$
\left|c_{n}\right| \leqq K l^{n}, \quad\left|a_{n-1}\right|+\frac{\left|b_{n}\right|}{\left|a_{n}\right|} \leqq k<l,
$$

For here we have

$$
u_{n} l^{-n} \rightarrow 0 .
$$

$$
\left|A_{n}^{r} l^{-n}\right| \leqq K(k / l)^{n-r}, \quad\left|B_{n}\right| \leqq B k^{n} .
$$

$\S 5$. We may rewrite the inequality (3) in the form

$$
\left|A_{n}^{r}\right| \leqq\left|a_{n} c_{r}\right| \prod_{s=r+1}^{n-1}\left[\frac{\left|b_{s+1}\right|}{\left|a_{s+1}\right|}\left(1+\frac{\left|a_{s} a_{s+1}\right|}{\left|b_{s+1}\right|}\right)\right]
$$

and deal similarly with the inequality for $\left|B_{n}\right|$. If we now impose suitable conditions on the infinite product

$$
\prod_{n=1}^{\infty} \frac{b_{n}}{a_{n}},
$$

theorems analogous to I, II, III above may be obtained. A formal statement of these is not, however, necessary here. 